\title{
Neurophotonics
}

\section{Alternating current and infrared produce an onset-free reversible nerve block}

Emilie H. Lothet

Kevin L. Kilgore

Niloy Bhadra

Narendra Bhadra

Tina Vrabec

Yves T. Wang

E. Duco Jansen

Michael W. Jenkins

Hillel J. Chiel 


\section{Alternating current and infrared produce an onset-free reversible nerve block}

\author{
Emilie H. Lothet, ${ }^{a}$ Kevin L. Kilgore, ${ }^{b, c, d}$ Niloy Bhadra, ${ }^{d}$ \\ Narendra Bhadra, ${ }^{d}$ Tina Vrabec, ${ }^{d}$ Yves T. Wang, ${ }^{d, e}$ \\ E. Duco Jansen, ${ }^{f}$ Michael W. Jenkins, ${ }^{\text {ed }}$ and \\ Hillel J. Chiel ${ }^{a, g, d, *}$ \\ ${ }^{\mathrm{a} C}$ Case Western Reserve University, Department of Biology, 2080 \\ Adelbert Road, Cleveland, Ohio 44106-7080, United States \\ ${ }^{b}$ MetroHealth Medical Center, Department of Orthopaedics, \\ Cleveland, Ohio 44109-1900, United States \\ 'Louis Stokes Cleveland Veterans Affairs Medical Center, Cleveland, \\ Ohio 44106-1702, United States \\ ${ }^{\mathrm{d}}$ Case Western Reserve University, Department of Biomedical \\ Engineering, Cleveland, Ohio 44106-7207, United States \\ ${ }^{e}$ Case Western Reserve University, Department of Pediatrics, \\ Cleveland, Ohio 44106-7207, United States \\ fVanderbilt University, Department of Biomedical Engineering, \\ Nashville, Tennessee 37235, United States \\ ${ }^{9}$ Case Western Reserve University, Department of Neuroscience, \\ Cleveland, Ohio 44106-7080, United States
}

\begin{abstract}
Nerve block can eliminate spasms and chronic pain. Kilohertz frequency alternating current (KHFAC) produces a safe and reversible nerve block. However, KHFAC-induced nerve block is associated with an undesirable onset response. Optical inhibition using infrared (IR) laser light can produce nerve block without an onset response, but heats nerves. Combining KHFAC with IR inhibition [alternating current and infrared (ACIR)] produces a rapidly reversible nerve block without an onset response. ACIR can be used to rapidly and reversibly provide onset-free nerve block in the unmyelinated nerves of the marine mollusk Aplysia californica and may have significant advantages over either modality alone. ACIR may be of great clinical utility in the future. $\odot$ The Authors. Published by SPIE under a Creative Commons Attribution 3.0 Unported License. Distribution or reproduction of this work in whole or in part requires full attribution of the original publication, including its DOI. [DOI: 10.1117/1.NPh.1.1.011010]
\end{abstract}

Keywords: nerve block; high-frequency alternating current; infrared laser light; Aplysia; pain; spasm; optical inhibition.

Paper 14020LSSR received Feb. 27, 2014; revised manuscript received May 19, 2014; accepted for publication Jul. 1, 2014; published online Jul. 28, 2014.

\section{Introduction}

Patients affected with neurological disorders are prone to chronic pain ${ }^{1}$ or spasmodic muscle contractions. ${ }^{2}$ Drugs or surgery can block undesirable neural activity; ${ }^{3,4}$ however, drugs have a slow time course and may have undesirable side effects, and surgery is usually irreversible. An ideal block would be fast and reversible over extended periods.

A promising new technology is kilohertz high-frequency alternating current (KHFAC), which reversibly blocks action potentials while still preserving nerve viability. ${ }^{5,6}$ KHFAC is

*Address all correspondence to: Hillel J. Chiel, E-mail: hjc@case.edu effective in vertebrate myelinated axons, ${ }^{5}$ and in unmyelinated axons from the marine mollusk Aplysia. ${ }^{7}$ Nerve block obtained with KHFAC is consistently associated with an initial "onset response," during which the nerve fires rapidly for milliseconds to seconds. The onset response may cause brief but intense muscle contractions and pain. To improve the clinical utility of KHFAC, the onset response should be eliminated.

Increasing neural temperature can induce action potential block. ${ }^{8}$ Modeling studies suggest that the block is due to altered ion channel kinetics. ${ }^{9}$ Studies show that infrared (IR) laser block is fast, reversible, and has high spatial resolution in rat myelinated nerves and in Aplysia unmyelinated nerves. ${ }^{10,11}$

We combined KHFAC and optical inhibition using IR lasers [alternating current and infrared (ACIR)] to produce fast and reversible nerve block without an onset response.

\section{Materials and Methods}

\subsection{Animal Preparation}

Unmyelinated nerves of Aplysia were used. Aplysia can be maintained for many hours and have previously been used to define appropriate parameters for optical block in myelinated rat sciatic nerve. ${ }^{10,11}$ Animals 300 to $400 \mathrm{~g}$ were used, as their nerves are 4 to $7 \mathrm{~cm}$, with a diameter of 0.5 to $1.5 \mathrm{~mm}$, comparable to rat sciatic nerve. Animals were anesthetized with isotonic magnesium chloride. The pleural-abdominal nerves were maintained in Aplysia saline ${ }^{12}$ at room temperature after dissection. All experiments were performed in vitro.

\subsection{Experimental Setup}

Suction electrodes and optical fibers were placed on the nerve (Fig. 1): a monopolar electrode for stimulation (a); 2 en passant electrodes for bipolar proximal recording (b); 2 optical fibers for IR block (c); 2 en passant electrodes for KHFAC block (d); and 2 electrodes for bipolar distal recording (e). En passant electrodes were chosen for convenience; cuff electrodes have previously been used successfully. Electrodes were filled with Aplysia saline before suctioning the nerve into the electrode to preserve nerve viability. $\mathrm{An} \mathrm{Ag} / \mathrm{AgCl}$ wire was inserted in each electrode. Electrical stimulation to generate nerve action potentials was controlled by a pulse generator (A310 Accupulser, WPI Instruments, Sarasota, Florida) via a stimulus isolator (WPI A360, WPI Instruments). KHFAC block was delivered by a controlled constantcurrent function generator (Model 6221, Keithley Instruments, Cleveland, Ohio) using a sinusoidal waveform. An inductor $(8.2 \mathrm{H})$ was placed across the function generator outputs to minimize DC leakage. The nerve compound action potential (CAP) was monitored using AxoGraph X (AxoGraph Scientific).

For IR block, 600- $\mu \mathrm{m}$ multimode optical fibers (P600-2-VISNIR, Ocean Optics, Dunedin, Florida) were placed on either side of the same nerve cross section to produce more uniform IR exposure. They were coupled to 2 Capella lasers (Lockheed Martin Aculight, Bothell, Washington) centered at 1860 and $1863 \mathrm{~nm}$ due to different wavelength tuning ranges, but with similar water absorption coefficients. The optical fibers gently touched the nerve sheath, producing a $600-\mu \mathrm{m}$ spot at the nerve surface. The fibers had a numerical aperture of 0.22 (i.e., a beam divergence of $25.4 \mathrm{deg}$ in air), so the spot was slightly larger at the axons due to divergence and scattering through the typically $100-\mu \mathrm{m}$ thick sheath. Since the onset response travels both anterogradely and retrogradely from the KHFAC block electrodes, 


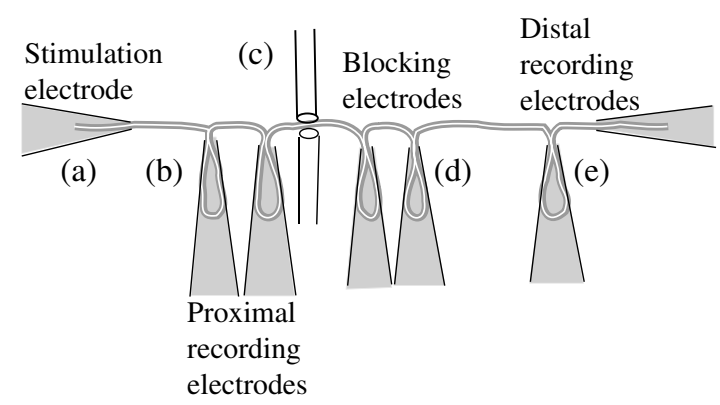

Fig. 1 Nerve preparation incorporating kilohertz high-frequency alternating current (KHFAC) and IR lasers. From left to right: stimulating electrode (a), proximal recording electrode (b), KHFAC blocking electrode (d), and distal recording electrode (e). Position of optical fibers between the proximal and blocking electrodes (c) are shown schematically (see text).

applying the lasers to block the onset response near the proximal electrodes allowed the distal electrodes to serve as a control for the same KHFAC block. Varying placement of the optical fibers between the KHFAC and the distal or proximal recording electrodes had no effect on the results.

\subsection{Experimental Protocol}

Three experiments were performed on three different nerves, using an A-B-A protocol. During protocol A, a train of action potentials was blocked by KHFAC; protocol B added IR inhibition to generate onset response block. Protocol A was repeated as a control. A current just above the stimulation threshold produced CAPs of sufficient amplitude to assess block effectiveness. The minimum amplitude KHFAC waveform at which block was observed was consistently at a frequency of $10 \mathrm{kHz}$ and amplitude ranging from 10 to $15 \mathrm{~mA}$ (peak-to-peak). For the two lasers, radiant exposures per pulse ranged from 0.177 to
$0.254 \mathrm{~J} / \mathrm{cm}^{2}$. Both lasers were switched on at the same time and emitted laser light for $30 \mathrm{~s}$ before the KHFAC waveform was applied, using 200- $\mu$ s pulses at $200 \mathrm{~Hz}$, to allow the temperature to reach a higher value. ${ }^{11}$ Nerve health was assessed before and after every experiment by comparing the propagating CAPs traveling down the length of the nerve.

\section{Results}

Figure 2 shows that application of KHFAC induced both an onset response (top panel and left box), and that it completely blocked the CAP (middle box), which returns after KHFAC was turned off (right box).

When only KHFAC is applied [Figs. 3(a) and 3(b), left panels], the onset response is visible in both the proximal and distal recordings. When IR is also applied, the onset response is blocked in the proximal recording only [Figs. 3(a) and 3(b), middle panels, shaded rectangles]. The onset response was still present at the distal recording because the onset response's propagation to that electrode was unaffected by the laser [Figs. 1(d) and 1(e)]. Although the onset response was present in the distal recording, the CAP was blocked by the laser [Fig. 3(c)]. The onset response reappeared proximally as soon as the laser was turned off [Figs. 3(a) and 3(b), right panels]. After ACIR, CAPs were triggered and had the same amplitude as before ACIR, demonstrating its reversibility. ACIR produced complete onset response block in each experiment $(N=3)$. The onset response, which has previously been shown to be variable over time, ${ }^{5}$ was not identical before, during, and after block, but the block of the onset response was always complete.

\section{Discussion}

Complete and reversible block without an onset response was obtained with ACIR. Previous studies using IR inhibition alone have found that the nerve physiology was unaffected by a similar protocol. ${ }^{10,11} \mathrm{We}$ also observed that the CAP

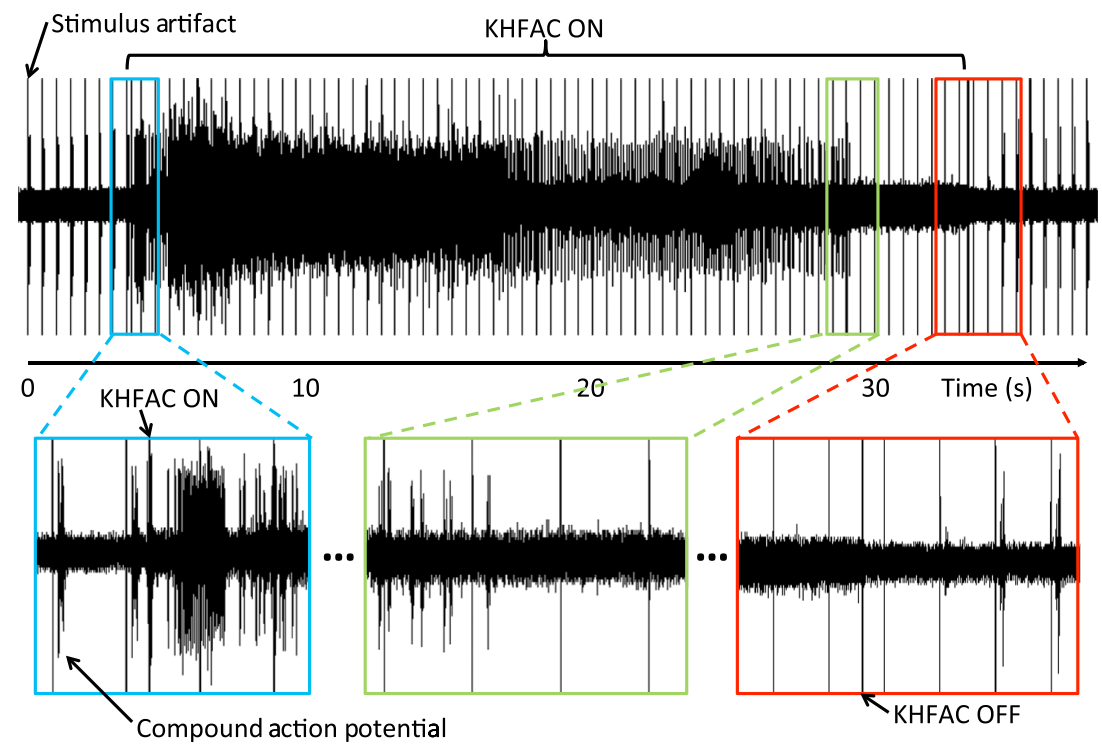

Fig. 2 KHFAC block and onset response as recorded through the distal recording electrode. Top trace (slower time scale) shows artifact from stimulating electrode at 500-ms intervals. When KHFAC is turned on, a long-lasting onset response is observed. Left box (faster time scale) shows the compound action potential (CAP) in response to stimulation, and the beginning of the onset response. Middle box shows that KHFAC has completely blocked the CAP after the onset response is over. Right box shows that CAP returns shortly after KHFAC is turned off. 


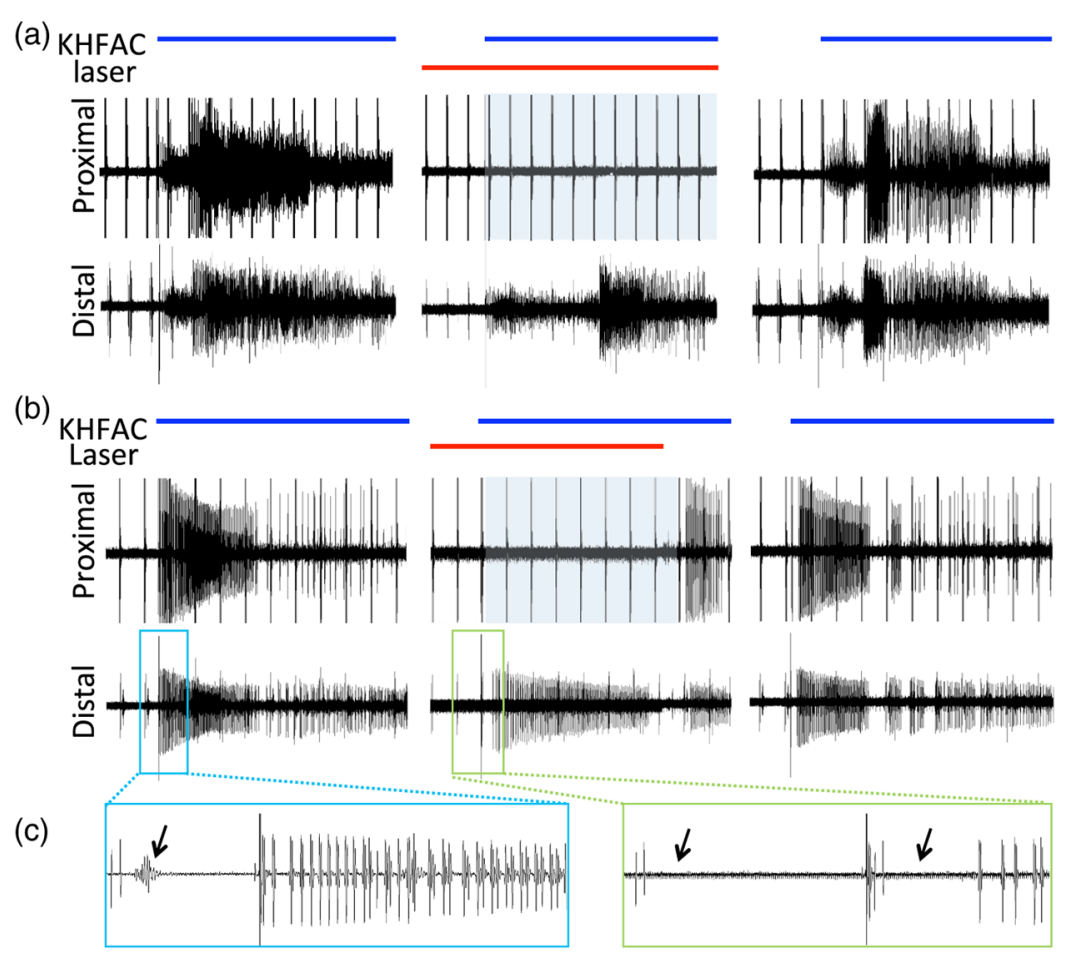

Fig. 3 Alternating current and infrared blocks onset response. (a) Left: Onset response due to KHFAC (top bar) in proximal (top) and distal (bottom) recording electrodes. Middle: (top) Complete suppression of onset response in proximal recording electrode after laser block (bar below top bar; block duration indicated by light shaded rectangle). The onset response continues in the distal recording (bottom). Right panels: Recovery of onset response in both proximal and distal recording electrodes when lasers are off. (b) A second experiment in a different animal. Middle top panel shows suppression of the onset response during laser block (bar below top bar; duration of onset response block indicated by light shaded rectangle). As soon as the laser is turned off, the onset response returns in the proximal recording. (c) (Left) Expanded time scale of outlined left box in part (b). Arrow indicates CAP occurring after the two spikes of the stimulation artifact. (Right) CAP is blocked in the distal recording by laser before and just after KHFAC is applied; arrows point to the times at which the CAP should appear.

was of similar size before and after treatment, suggesting that the nerve's physiology was unaffected. Future studies will be necessary to determine if chronic exposure to this treatment has a deleterious effect on nerves. It will be critical to establish the safety of different optical protocols.

Preliminary studies have indicated that a higher radiant exposure can lead to optical inhibition within $100 \mathrm{~ms}$. A continuous wave laser may be more efficient than the pulsed lasers with $4 \%$ duty cycles used in this study. ACIR is a promising method of providing fast reversible block and may have application in the treatment of chronic pain and muscle spasticity.

\section{Acknowledgments}

Research was supported by NSF grant DMS-101043, NIH grants R21-HL115373, R01-NS-074149, and R01-NS052407, and DOD grant (W81XWH-10-C-0208).

\section{References}

1. R. H. Dworkin et al, "Interpreting the clinical importance of treatment outcomes in chronic pain clinical trials: IMMPACT recommendations," J. Pain 9(2), 105-121 (2008).

2. J. W. Little and T. L. Massagli, "Spasticity and Associated Abnormalities of Muscle Tone," in Rehabilitation Medicine Principles and Practice, 3rd ed., J. A. DeLisa and B. M. Gans, eds., Lippincott-Raven Publishers, Philadelpha, PA (1998).
3. J. M. Gracies et al., "Traditional pharmacological treatments for spasticity part I: Local treatments," Muscle Nerve Suppl. 20(Supplement 6), 61-91 (1997).

4. J. M. Gracies et al., "Traditional pharmacological treatments for spasticity. Part II: General and regional treatments," Muscle Nerve Suppl. 20, S92-S120 (1997).

5. K. L. Kilgore and N. Bhadra, "Reversible nerve conduction block using kilohertz frequency alternating current," Neuromodulation 17(3), 242255 (2014).

6. K. L. Kilgore and N. Bhadra, "Nerve conduction block utilising highfrequency alternating current," Med. Biol. Eng. Comput. 42(3), 394-406 (2004).

7. L. Joseph and R. J. Butera, "Unmyelinated Aplysia nerve exhibit a nonmonotonic blocking response to high-frequency stimulation," IEEE Trans. Neural Sys. Rehab. Eng. 17(6), 537-544 (2009).

8. A. L. Hodgkin and B. Katz, "The effect of temperature on the electrical activity of the giant axon of the squid," J. Physiol. 109(1-2), 240-249 (1949).

9. Z. Mou et al., "A simulation study of the combined thermoelectric extracellular stimulation of the sciatic nerve of the Xenopus laevis: the localized transient heat block," IEEE Trans. Biomed. Eng. 59(6), 1758-1769 (2012).

10. A. R. Duke et al., "Spatial and temporal variability in response to hybrid electro-optical stimulation," J. Neur. Eng. 9(3), 036003 (2012).

11. A. R. Duke et al., "Transient and selective suppression of neural activity with light," Sci. Rep. 3, 2600 (2013).

12. J. M. McManus, H. Lu, and H. J. Chiel, "An in vitro preparation for eliciting and recording feeding motor programs with physiological movements in Aplysia californica," J. Vis. Exp. (70), e4320 (2012). 